International Journal of Research in Engineering and Innovation
(IJREI)
journal home page: http://www.ijrei.com
ISSN (Online): 2456-6934

\title{
Various analytical methods for the determination of clarithromycin- A review
}

\author{
Sarra A. Abrahem¹, Abdul kader S², Suhad A. Ibrahim ${ }^{1}$ \\ ${ }^{I}$ Department of Chemistry, College of Science at Al-Nahrain University, Baghdad, Iraq \\ ${ }^{2}$ Minister's office, Ministry of higher education and scientific research, Baghdad, Iraq.
}

\begin{abstract}
Clarithromycin has a broad spectrum of antibacterial properties. It is considered safe and necessary in any health system according to the World Health Organization's Essential Medicines List. Therefore, it is important to develop simple and low-cost analytical methods for these compounds in order to obtain a better quality control. This paper reviews a number of analytical papers that identify clarithromycin in commercial preparations and biological samples, and these include chromatography, ion selective electrodes and spectrophotometric.

Keywords: Clarithromycin, ISE, HPLC, Spectrophotometric

\section{Introduction}

Clarithromycin is a type of medicine available as a generic medicine and is beneficial in treating some bacterial infections such as Helicobacter pylori, and pneumonia and it is also used as a penicillin substitute for cycloid bacteria.(1) It is an antibiotic that has good stability in stomach acid and its pharmacological profile is more suitable than erythromycin for semisynthetic macrolide.(2) Clarithromycin's chemical structure, as shown in figure 1, has the empirical formula $\left(\mathrm{C}_{38} \mathrm{H}_{69} \mathrm{NO}_{13}\right)$. It is almost crystalline, white powder with a molecular weight $748 \mathrm{~g} / \mathrm{mole}$, soluble in acetone, slightly dissolved in methanol, meanwhile insoluble in water.(3) To define and study clarithromycin, various analytical methods were used, and studied: limit of detection, concentration range, recovery, life time, type of column, mobile phase, slope, retention time and the range of $\mathrm{PH}$ for clarithromycin. The results were listed in Tables 1, 2, and 3.

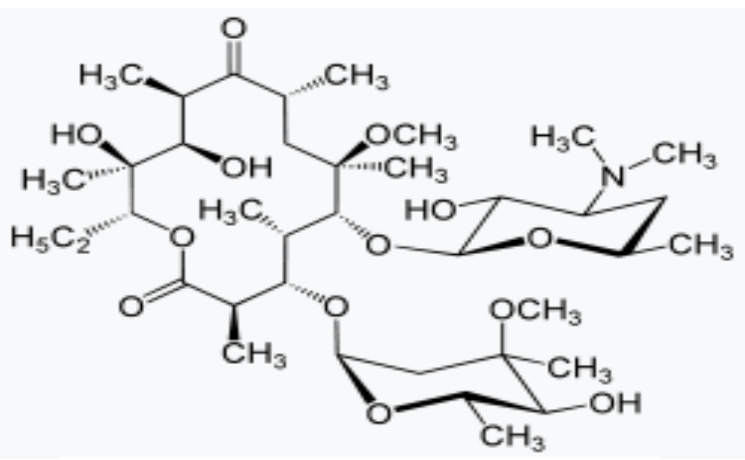

(a)

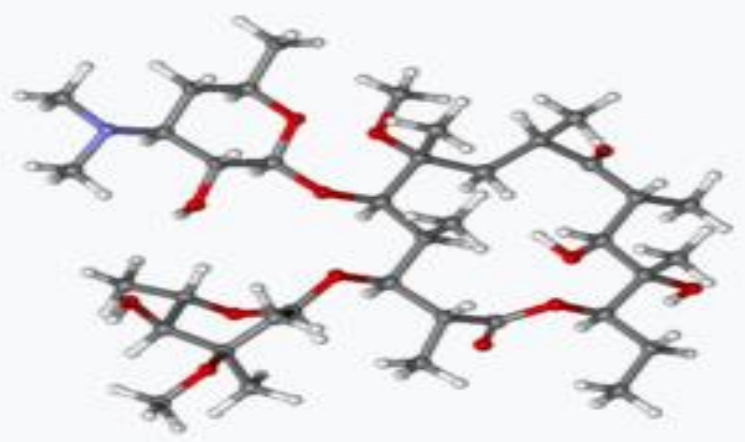

(b)

Figure 1: (a) 2D chemical structures of clarithromycin, (b) $3 D$ chemical structures of clarithromycin

Corresponding author: Suhad A Ibrahim

Email Address: su_aziz2015@yahoo.com 
Table 1: HPLC for determination of clarithromycin

\begin{tabular}{|c|c|c|}
\hline Method & results & Ref. \\
\hline RP-HPLC & $\begin{array}{l}\text { Column: A }(5 \mu \mathrm{m}) \text { ODS Kromasil, } 250 \times 4.6 \mathrm{~mm} \\
(\mathrm{C} 18 \text { column). } \\
\text { Mobil Phase: Contains a }(0.05 \mathrm{M}) \text { phosphate buffer and }(0.005 \mathrm{M}) \\
\text { sulphonic acid sodium salt mono-hydrate of }(\mathrm{pH} \mathrm{3.2).} \mathrm{For} \mathrm{separati} \\
\text { quantification, }(50: 50 \mathrm{v} / \mathrm{v}) \text { acetonitrile mixture was used. } \\
\text { Detector: UV detector. } \\
\text { Conc. range: } 75-175 \mu \mathrm{g} / \mathrm{mL} \text {. } \\
\text { LOD: } 5.230 \mu \mathrm{g} / \mathrm{mL} \text {. } \\
\lambda: 205 \mathrm{~nm} \text {. } \\
\text { tR: } 2.21 \mathrm{~min}\end{array}$ & [4] \\
\hline RP-HPLC & $\begin{array}{l}\text { Column: In an isocratic mode, } 5 \mu \mathrm{m} \text { particle size, } 150 \mathrm{~mm} \text { length } \times 4.6 \\
\text { mm i.d (C18 column). } \\
\text { Mobil Phase: composed of acetonitrile in a ratio of }(55: 45 \text {, v/v).and } \\
0.035 \mathrm{M} \text { potassium dihydrogen phosphate }(\mathrm{pH} 4.4 \pm 0.017) \\
\text { Detector: UV detector. } \\
\text { Conc range: } 320-480 \mu \mathrm{g} / \mathrm{ml} \text {. } \\
\text { LOD: } 0.04 \mu \mathrm{g} / \mathrm{mL} \text {. } \\
\lambda: 210 \mathrm{~nm} \text {. } \\
\mathbf{t}_{\mathbf{R}}: 4.100 \pm 0.074 \mathrm{~min} .\end{array}$ & [5] \\
\hline RP-HPLC & $\begin{array}{l}\text { Column: } 250 \times 4.6 \mathrm{~mm}, 5 \mu \mathrm{m},(\mathrm{C} 8 \text { column }) \text {. } \\
\text { Mobil Phase: Consists of acetonitrile and dipotassium hydrogen } \\
\text { orthophosphate buffer }(0.1 \mathrm{M}) \text { of }(\mathrm{pH} 6.0) \text { in a ratio of } 50: 50(\% \mathrm{v} / \mathrm{v}) \text {. } \\
\text { Detector: UV detector. } \\
\text { Conc range: } 4.0-5000.0 \mu \mathrm{g} / \mathrm{ml} \text {. } \\
\text { LOD: } 4.0( \pm 6.6 \%) \text {. } \\
\lambda: 210 \mathrm{~nm} \text {. }\end{array}$ & [6] \\
\hline Liquid Chromato-graphic & $\begin{array}{l}\text { Column: Shim pack CLC-ODS column. } \\
\text { Mobil Phase: a mixture of phosphate buffer }(0.05 \mathrm{M}) \text { that contain a } \\
(17: 83, \mathrm{v} / \mathrm{v}) \text { methanol and }(2 \mathrm{ml} / \mathrm{l} ; \mathrm{pH} 3.8) \text { triethylamine. } \\
\text { Detector: fluorescence detector. } \\
\text { Conc range: } 0.025-10 \mu \mathrm{g} / \mathrm{ml} \text {. } \\
\lambda: 265 \mathrm{~nm} \text {. }\end{array}$ & [7] \\
\hline HPLC & $\begin{array}{l}\text { Column: Shodex Asahipak high-performance liquid chromatography } \\
\text { (HPLC) column. } \\
\text { Mobil Phase: it contain acetonitrile and phosphate buffer with the ratio } \\
\text { of (pH } 11 \pm 0.05) \text { of }(60: 40, \mathrm{v} / \mathrm{v}) \text {. } \\
\text { Detector: the type is UV detector. } \\
\text { LOD: from } 214 \text { to } 228 \mu \mathrm{g} / \mathrm{kg} \text {. } \\
\lambda: 210 \mathrm{~nm} \text {. }\end{array}$ & [8] \\
\hline $\begin{array}{l}\text { RP-LC with Electrochemical } \\
\text { Detection }\end{array}$ & $\begin{array}{l}\text { Column: at } 408{ }^{\circ} \mathrm{C}, \mathrm{C} 8 \text { column }(5-\mathrm{mm}(10034.6 \mathrm{~mm}) \text {. } \\
\text { Mobil Phase: Acetonitrile with }(37: 63, \mathrm{v} / \mathrm{v}) 0.045 \mathrm{M} \mathrm{H} 3 \mathrm{PO} 4 \text { and }(\mathrm{pH} \\
6.7) \text {. } \\
\text { Detector: The electrochemical detector, L-ECD-6A, operates at }(0.85 \mathrm{~V}) \\
\text { level, using a glass carbon electrode with a reference electrode } \\
(\mathrm{Ag} / \mathrm{AgCl}) . \\
\text { Conc range: } 0.05-5.0 \mathrm{mg} / \mathrm{mL} \text {. } \\
\text { LOQ: } 0.05 \mathrm{mg} / \mathrm{mL} . \\
\lambda: \text { below } 220 \mathrm{~nm} . \\
\mathbf{t}_{\mathbf{R}}: 12.26 \mathrm{~min} .\end{array}$ & [9] \\
\hline HPLC & $\begin{array}{l}\text { Column: C8 Lichrosorb TM analytical column, }(150 \mathrm{~mm} \times 4.6 \mathrm{~mm} \text {, } \\
10 \mu \mathrm{m}) \text {. } \\
\text { Mobil Phase: The mixture in ratio }(33: 17: 50, \mathrm{v} / \mathrm{v} / \mathrm{v}),(\text { acetonitrile, } \\
\text { methanol, phosphate buffer) at } \mathrm{pH} 3.5 \\
\text { Detector: UV detector. } \\
\text { Conc range: } 75-2000 \mu \mathrm{g} \mathrm{mL}^{-1} \text {. } \\
\text { Coefficient of variation: } 0.862 \text {. } \\
\lambda: 210 \mathrm{~nm} \text {. } \\
\mathbf{t}_{\mathbf{R}}: 5.44 \mathrm{~min} \text {. }\end{array}$ & [10] \\
\hline
\end{tabular}




\begin{tabular}{|c|c|c|}
\hline RP-HPLC & $\begin{array}{l}\text { Column: Waters Spheris orb } 5 \mu \mathrm{m} \text { ODS2, } 4.6 \times 250 \mathrm{~mm} \text { (C18 column). } \\
\text { Mobil Phase: Pairing reagent (Acetonitrile/aqueous trifluoroacetic acid). } \\
\text { Detector: SEDERE - ELS Detector (SEDEX MODEL 75). } \\
\text { Conc range: } 5-100 \mu \mathrm{g} / \mathrm{mL} \text {. } \\
\text { LOD: } 1.6 \mathrm{mg} / \mathrm{L} . \\
\mathbf{t}_{\mathbf{R}}: 4.7 \mathrm{~min} .\end{array}$ & [11] \\
\hline $\begin{array}{l}\text { Pre-column derivatization } \\
\text { with HPLC-UV method }\end{array}$ & $\begin{array}{l}\text { Column: } \mathrm{C}_{18} \text { column. } \\
\text { Mobil Phase: }(50 \mathrm{mM}, \mathrm{pH} 6.8,0.7 \% \text { triethylamine }) \text { potassium } \\
\text { dihydrogen phosphate, }(30: 45: 25, \mathrm{v} / \mathrm{v} / \mathrm{v}) \text {, and methanol acetonitrile. } \\
\text { Detector: UV detector. } \\
\text { Conc range: } 0.1-10 \mu \mathrm{ml}^{-1} \text {. } \\
\text { LOD: } 30 \mathrm{ng} \mathrm{ml}{ }^{-1} \text {. } \\
\lambda: 275 \mathrm{~nm} \text {. }\end{array}$ & [12] \\
\hline
\end{tabular}

Table 2: Ion-Selective Electrodes for Determination of clarithromycin

\begin{tabular}{|c|c|c|}
\hline $\begin{array}{c}\text { Type of Ion-pair for } \\
\text { Electrodes }\end{array}$ & Results & Ref. \\
\hline $\begin{array}{l}\text { Clarithromycin } \\
\text { phosphotun-gestate } \\
\text { (CLR-PTA) }\end{array}$ & $\begin{array}{l}\text { Conc. Range: } 10^{-6} \text { to } 10^{-2} \mathrm{~mol} / \mathrm{L} \text {. } \\
\text { Plasticizer: dioctylphthalate (DOP). } \\
\text { Slope: } 58.8 \mathrm{mV} / \text { decade. } \\
\text { pH range: } 3-8 \text {. } \\
\text { Detection limit: } 2.5 \times 10-6 \mathrm{~mol} . \mathrm{L}^{-1} \text {. } \\
\text { Correlation coefficient }(\mathbf{r}): 0.9999 \text {. } \\
\text { Life time: } 30 \text { days. }\end{array}$ & [13] \\
\hline $\begin{array}{l}\text { Clarithromycin- } \\
\text { tetraphenyl-borate ion } \\
\text { pair complex }\end{array}$ & $\begin{array}{l}\text { Conc. Range: } 1 \times 10^{-5}-1 \times 10^{-3}, 1 \times 10^{-5}-1 \times 10^{-3}, 5 \times 10^{-5}-1 \times 10-3 \text { and } 1 \times 10^{-5}-1 \times 10^{-3} \\
\text { M) respectively. } \\
\text { Plasticizers: Di-octyl phthalate (DOP); Di-butyl phosphate (DBP); } \\
\text { Acetophenone (AP); Di-butyl phthalate (DBPH). } \\
\text { Slope: } 51.206,53.930,58.104 \text { and } 58.484 \mathrm{mV} / \text { decade). } \\
\text { Detection limit: } 8 \times 10^{-6}, 6 \times 10^{-6}, 2 \times 10^{-5} \text { and } 9 \times 10^{-6} \text {. } \\
\text { Life time: } 24,12,45 \text { and } 20 \text { days. }\end{array}$ & [3] \\
\hline
\end{tabular}

Table 3: Spectrophotometric methods for determination of clarithromycin

\begin{tabular}{|c|c|c|}
\hline Methods & results & Ref. \\
\hline $\begin{array}{c}\text { Derivative } \\
\text { Spectrophoto-metric }\end{array}$ & $\begin{array}{l}\text { Sample: Clarithromycin. } \\
\text { Conc range: } 5.0 \text { to } 60 \mu \mathrm{g} \mathrm{mL}-1 \text {. } \\
\text { Slope: } 0.00625 \text {. } \\
\mathbf{r}^{2}: 0.9998 . \\
\text { LOD: } 1.055 \mu \mathrm{g} / \mathrm{mL} \text {. } \\
\lambda_{\max }: 268 \mathrm{~nm} .\end{array}$ & [14] \\
\hline $\begin{array}{l}\text { UV Spectrophoto- } \\
\text { metric }\end{array}$ & $\begin{array}{l}\text { Sample: terbinafine hydrochloride and clarithromycin (TRB, CAM). } \\
\text { Conc. range: a TRB: } 0.5-3.0 \mathrm{ml}, 50 \mu \mathrm{g} \cdot \mathrm{ml}^{-1} \text {; CAM: } 0.5-3.0 \mathrm{ml}, 100 \mu \mathrm{g} \cdot \mathrm{ml}^{-1} \text {. } \\
\text { Slope: }\left(3.013 \times 10^{-2}, 1.829 \times 10-2\right) \text {. } \\
\mathbf{r}^{2}:(0.9999,0.9999) \\
\text { LOD: }\left(1.299 \times 10^{-1}, 0.2737\right) . \\
\lambda_{\max }: 350 \mathrm{~nm} .\end{array}$ & {$[15]$} \\
\hline U.V. spectroscopy & $\begin{array}{l}\text { Sample: Clarithromycin. } \\
\text { Conc range: } 2-40 \mu \mathrm{g} / \mathrm{ml} \\
\text { Slope: } 0.63 \text { at } \mathrm{pH}=1.2 \\
\mathbf{r}^{2}: 0.9808 . \\
\text { LOD: } 1.242 \mu \mathrm{g} / \mathrm{ml} \\
\lambda_{\max }: 353 \mathrm{~nm}\end{array}$ & {$[16]$} \\
\hline Spectrophoto-metric & $\begin{array}{l}\text { Sample: Clarithromycin. } \\
\text { Conc range: } 10-70 \mu \mathrm{g} \cdot \mathrm{ml}^{-1} \text {. } \\
\text { Slope: } 0.0089 . \\
\mathbf{r}^{2}: 0.9999, \lambda_{\max }: 600 \mathrm{~nm} .\end{array}$ & [16] \\
\hline $\begin{array}{l}\text { UV Spectrophoto- } \\
\text { metric }\end{array}$ & $\begin{array}{l}\text { Sample: Clarithromycin. } \\
\text { Conc.: } 100 \mathrm{ppm} \text { in } 100 \mathrm{ml} \text {. } \\
\lambda_{\max }: 210 \mathrm{~nm} .\end{array}$ & {$[17]$} \\
\hline
\end{tabular}




\begin{tabular}{|c|c|c|}
\hline $\begin{array}{l}\text { UV Spectrophoto- } \\
\text { metric }\end{array}$ & $\begin{array}{l}\text { Sample: clarithromycin. } \\
\text { Conc. range: } 20-120 \mu \mathrm{g} / \mathrm{mL} \text {. } \\
\text { Slope: } 0.012 . \\
\mathbf{r}^{2}: 0.9997 . \\
\lambda_{\max }: 760.5 \mathrm{~nm} . \\
\end{array}$ & [18-19] \\
\hline Spectrophoto-metric & $\begin{array}{l}\text { Sample (1): (Clarithromycin and bromothymol blue (BTB)). } \\
\text { Sample (2): (Clarithromycin and bromocresol purple (BCP)). } \\
\text { Conc range: BTB } 0.5-25.0 \mu \mathrm{g} / \mathrm{ml} \text { and BCP } 1.0-25.0 \mu \mathrm{g} / \mathrm{ml} \text {, } \\
\text { Slope: } 0.0232 \text { and } 0.0322 \text { for BTB and BCP respectively. } \\
\mathbf{r}^{2}: 0.9998 \text { and } 0.9997 \text { for BTB and BCP. } \\
\text { LOD: } 0.36 \mu \mathrm{g} / \mathrm{ml} \text { and } 0.24 \mu \mathrm{g} / \mathrm{ml} \text {, for BTB and BCP. } \\
\lambda_{\text {max }} 416 \mathrm{~nm} \text { and } 418 \mathrm{~nm} \text { for BTB and BCP. }\end{array}$ & [19] \\
\hline $\begin{array}{l}\text { Visible Spectrophoto- } \\
\text { metric }\end{array}$ & $\begin{array}{l}\text { Sample (1): (clarithromycin, } \mathrm{HCl} \text {, acetone). } \\
\text { Conc. range: } 50-500 \mu \mathrm{g} / \mathrm{mL} \text {. } \\
\lambda_{\max } \text { } 485 \mathrm{~nm} . \\
\text { Sample }(\mathbf{2}, \mathbf{3}):(\text { clarithromycin, bromocresol green, bromopbnol blue). } \\
\text { Conc. range: } 0-60 \mu \mathrm{g} / \mathrm{mL} \text {. } \\
\lambda_{\max } 414 \mathrm{~nm} .\end{array}$ & {$[20]$} \\
\hline $\begin{array}{c}\text { UV visible } \\
\text { spectrophoto-meter }\end{array}$ & $\begin{array}{l}\text { Sample: Clarithromycin } \\
\text { Conc range: } 10-50 \mu \mathrm{g} / \mathrm{ml} \text {. } \\
\mathbf{r}^{2}: 0.990 \\
\lambda_{\max }: 416 \mathrm{~nm}\end{array}$ & {$[17]$} \\
\hline
\end{tabular}

\section{Conclusions}

In Table 1, 2, and 3 diverse theoretical studies of three analytical methods for calculating clarithromycin were included. It turns out from the study that, the best way to calculate clarithromycin in pharmaceutical preparations is (HPLC) high-performance liquid chromatography method. This method gave a wide range of concentration that was specified in $\mathrm{ng} / \mathrm{ml}$ and $\mu \mathrm{g} / \mathrm{ml}$, as well as low detection limit plus the most commonly used solvents are acetonitrile and methanol. Nonetheless, spectrophotometric and ion selective electrodes, can be described as easy to use methods and applications for calculating clarithromycin in pharmaceutical samples in addition to their low coast in quality control analysis compounds in pharmaceutical preparations.

\section{References}

[1] Al-Saidi KH, Munshid HQ. New Polymeric Membrane Electrode for Clarithromycin Determination. J Al-Nahrain Univ Sci [Internet]. 2013 Jun $1 ; 16(2): 30-6$.

[2] Fotoohi K, Skärby T, Söderhäll S, Peterson C, Albertioni F. Interference of 7-hydroxymethotrexate with the determination of methotrexate in plasma samples from children with acute lymphoblastic leukemia employing routine clinical assays. J Chromatogr B Anal Technol Biomed Life Sci. 2005;817(2):139-44.

[3] Gangishetty S, Verma S. RP-HPLC Method Development and Validation for Simultaneous Estimation of Clarithromycin and Paracetamol. 2013;2013.

[4] Alam M, Hossain S, Bhadra S, Kumar U, Shara A, Rouf S. Development and Validation of RP-HPLC Method for Quantitation of Clarithromycin in Matrix Tablet Dosage Form. 2017;2(1):69-75.

[5] Katsidzira RM, Wessels A, Aucamp M. A novel rp-hplc method for the detection and quantification of clarithromycin or spiramycin in bulk drug samples and dosage forms. Int J Pharm Pharm Sci. 2016;8(12):310-3.
[6] Farshchi A, Ghiasi G, Bahrami G. A sensitive liquid chromatographic method for the analysis of clarithromycin with pre-column derivatization: Application to a bioequivalence study. Iran J Basic Med Sci. 2009;12(1):2532.

[7] Zaater MF, Tahboub YR, Ghanem E. Determination and stability assessment of clarithromycin in human plasma using RP-LC with electrochemical detection. J Chromatogr Sci. 2012;50(9):763-8.

[8] Salem H, Salem H, Riad M, Reda M, Ahmed K. Simultaneous determination of omeprazole, tinidazole and clarithromycin in bulk powder and Helicure tablets by TLC-densitometric technique Simultaneous determination of omeprazole, tinidazole and clarithromycin in bulk powder and Helicure tablets by TLC. 2016;(October).

[9] Tzouganaki Z, Koupparis M. Development and validation of an HPLC method for the determination of the macrolide antibiotic clarithromycin using evaporative light scattering detector in raw materials and pharmaceutical formulations. Mediterr J Chem. 2017;6(4):133-41.

[10] Li W, Jia H, Zhao K. Determination of clarithromycin in rat plasma by HPLC - UV method with pre-column derivatization. 2007;71:385-90.

[11] Ibrahim SA, Abrahem SA. Molecular identification of heterocyclic compound-phenol degrading bacteria from industrial soil. 2015. 2015;2741.

[12] Abrahem SA, Abass AM, Ahmed A. Trimethoprim determination with drug-selective electrodes. 2019;12(6).

[13] Riad S, Rezk M, Ahmed K, Salem H. Analytical \&. 2014;(December).

[14] Hassan W, Zaman B, Ali B. Derivative spectrophotometric method for the determination of clarithromycin in pharmaceutical formulation and application to in-vitro dissolution . 2018;2(1):1-8.

[15] Rm R, Csp S. Modern Chemistry \& Applications UV Spectrophotometric Analysis of Drugs Terbinafine Hydrochloride and. 2018;6(2):1-5.

[16] uv15.pdf.

[17] Naveed S, Qamar F. Simple UV Spectrophotometric Assay of Metronidazole. OALib. 2014;01(06):1-4.

[18] Desai V, Sharma S, Patil N. Alternative Medicines and Their Applications in Dentistry- A Brief Review. 2013;2(2):542-5.

[19] Tadros T. Pharmaceutical Formulations. Encycl Colloid Interface Sci. 2013;41(2):910-1.

[20] visible-spectophotometric-methods-for-estimation-of-clarithromycin-fromtablet-formulation.pdf.

Cite this article as: Sarra A. Abrahem, Abdul kader S, Suhad A. Ibrahim, Various Analytical Methods for the Determination of Clarithromycin -AReview, International Journal of Research in Engineering and Innovation Vol-4, Issue-1 (2020), 56-59. https://doi.org/10.36037/IJREI.2020.4105 